Carsten S. Østerlund

\title{
Documenting Practices
}

\section{The indexical centering of medical records}

\begin{abstract}
This paper explores how organizational members use documents to share their knowledge within and across work settings. I suggest that organizational studies of distributed knowledge sharing and information systems would greatly benefit from the linguistic analysis of communicative practices. Specifically, the paper highlights the notion of indexical centering as formulated by the linguistic anthropologist William Hanks and demonstrates its analytical power in studying documenting as a communicative practice. Drawing on a 15-month, multisited ethnographic study in several pediatric healthcare settings, the paper focuses on how two doctors compose and use two medical histories found in two distinct medical information systems. The analysis suggests that the doctors use documents to index the temporal, spatial, and participatory dimensions of their knowledge sharing. They do so by indexing, on the one hand, the participants, times and places for their communicative practices and, on the other hand, the participants, times and places of their general care practices. The indexical analysis allows us to perceive documents, as more than mere vessels for knowledge transfer among organizational members, but as an integrated part of how people structure their work practices and situate their knowledge sharing in complex distributed organizational settings.
\end{abstract}

\section{Introduction}

\section{Vignette: Two patients, two doctors, two information systems}

Around four o'clock on a February afternoon in Kiltham Hospital an infant boy, Dylan, lies in a small transparent plastic crib. Two doctors and a medical student are simultaneously leaning over Dylan, three stethoscopes pressed to his chest listening, eyes turned to the ceiling. The medical student and two doctors, an intern and a senior resident, finish their exam and turn to the other infant in the room, Anna. Similar to Dylan, she has been admitted for bronchiolitis. Both infants spent several weeks in the hospital, first in the intensive care unit (ICU) and then transferred to their current beds in a regular pediatric department, 10 East. The intern, Marc, a newly minted doctor in his first year of medical residency, and the senior resident, Elisabeth, in the fourth year of her residency, turn to Anna's mother sitting weary-looking beside Anna's crib.

Elisabeth says, "We know this has been a long ordeal for all of you; but we think Anna will be ready to go home tomorrow or the day after." Marc continues: "I will put the discharge papers together and the nurse will help you get ready to go home." 
After they have assured Anna's mother that her baby will be fine, Marc, Elisabeth, and the medical student all head for the doctors' conference room in this medical unit. The medical student grabs a clean Progress Note sheet at the nursing station. Behind the glass walls, known as the aquarium, Marc, the intern, and Elizabeth, the senior resident, each find a computer terminal. Marc logs on to the "House Officer Sign-Out" (HOSO), an on-line system. Elisabeth logs into the senior resident note system. They each start updating their notes on Dylan, Anna and the other patients they have seen since 7:00am. Marc will never read the senior resident's notes and vice-versa. Neither of these documents go into the official medical record nor do Marc and Elisabeth's supervisors access those two information systems to evaluate them or compensate them for their work.

\section{Information systems and knowledge sharing}

At first glance it seems counterintuitive if not counterproductive that the senior resident and the intern would not use the same information system to document their care. Elisabeth spends most of the day in close collaboration with Marc and three other interns. They gather for rounds in the morning, see new patients together, go to radiology rounds, have noon conferences, and share meal breaks. In the afternoon the senior works closely with one or more interns in the team's conference room writing notes or going to patient rooms for joint interviewing and patient examination. As in Dylan and Anna's cases, it is not uncommon to see a medical student, an intern, and a senior resident all bent over the same child, each with their stethoscopes on the young patient's chest.

These groups obviously share practices, they regard themselves as teams, yet they do not share the same document genres. Marc documents Dylan and Anna's histories in the HOSO, and Elisabeth, the senior resident, documents it in the Senior Notes. These differences are particularly apparent during morning rounds when interns and senior residents can be seen equipped with starkly different types of documents, each describing the same patients in slightly different formats. The interns shuffle through long printouts from their HOSO online system which lists all the patients seen by the team in alphabetic order including important information on problems, medications, and tests. In contrast, the senior resident on the team holds a printout neatly stapled together with small concise narratives summarizing individual patient cases.

One can observe comparable documenting practices among the nurses and other physicians involved in Dylan and Anna's care. Each healthcare provider typically maintains multiple records of patient care, many of which they do not share with other collaborators. Such observations irk the medical informatics community which has worked for the past three decades to develop universal patient-centered records - placing all relevant information about a patient's history at doctors' and nurses' fingertips. Researchers in the American Medical Informatics Association's (AMIA) Work Group for People and Organizational Issues (poiwg@mail.amia.org) regularly have list server discussions on the topic of failure rates in healthcare information systems (IS). Though impossible to verify, many quote $80 \%$ failure rates for the implementation of medical information systems. The exact percentage aside, today one finds that individual settings, departments, and sub-disciplines within healthcare facilities have implemented their own information systems. For instance, emergency departments will often have one electronic record system, the Intensive Care Unit (ICU) another, outpatient care a third, and nurses (in some hospitals) yet another nurse-use-only online record system; rarely do these systems communicate. 
The problem speaks to a larger theoretical question of how people use documents and information systems to coordinate their activities and knowledge about patients within and across settings. More generally, the question becomes: how do organizations best support viable information systems that sustain their members' capabilities to operate effectively both within and across temporally and geographically distributed settings? In recent years, this issue has received increased attention in the management and organization studies literature with the proliferation of distributed work organizations, virtual teams, and various information technologies attempting to support organizational structures and the sharing of knowledge among its members.

A special issue of Organization Science on knowledge illustrates this debate (Grandori et al, 2002) and its general push to differentiate types of knowledge to account for the sharing of knowledge in various organizational settings. As Orlikowski (2002) points out, this body of literature differentiates at least two types of knowledge, one explicit and abstract, and the other situated. For instance, Polanyi's (1983) distinction between "tacit" and "explicit" knowing is often used to characterize two types of knowledge or justify related dichotomies, such as "local" versus "universal", "know-how" versus "know-that", "formal" versus "situated", "canonical" versus "noncanonical” (Orlikowski, 2002: 253). Each of these conceptual pairs draws on different literatures and stresses unique theoretical points. However, if we glance over these individual variations we find an overarching dichotomy cutting across these conceptual pairs.

One pole treats knowledge as abstract representations, a perspective that has informed studies of managerial cognition (Walsh, 1995; Walsh \& Ungson, 1991). In the medical field this would be the abstracted, explicitly represented and codified knowledge taught in medical schools. The other pole approaches knowledge as local, context dependent, and emerging from interactions and practices in particular contexts. This would be the knowledge involved in the practice of medicine within specific healthcare settings, given changing collaborators and unfolding care for particular patients. One should note that "situated" typically gets depicted as "local" or context bound. The dichotomy creates a divide between abstract transferable knowledge and situated non-transferable knowledge.

From this perspective, situated knowledge cannot be shared across contexts. Situated knowledge becomes not only embedded in a context but bound to a context (Dreier, 1999). In other words, if one remains locked in this abstract/situated dichotomy it becomes impossible to develop a situated perspective that takes into account the sharing of situated knowledge across contexts in complex distributed organizational settings. In the following discussion I will use "abstract versus situated" as shorthand for this broader dichotomy.

Such a polarizing approach to knowledge is reflected in the conceptualization of documents and information systems. Documents are often depicted as containers for abstract, formal, homogeneous knowledge that can be easily transported across settings. In turn, these containers are not capable of capturing and disseminating local, messy, heterogeneous, and concrete knowledge. Taking a step back, one could argue that this framework addresses the question raised above, whether people can share situated knowledge beyond the context in which it is embedded. And, the answer is no. People share abstract codified knowledge - not situated and contextually embedded knowledge.

In other words, documents and the knowledge represented in them are pictured as hovering above the realm of the empirical and contextual. Two opposing discourses about the organizational role of documents and information systems easily follow (Berg, 1997a). On 
one hand, we find the position with the power of information systems and formal tools residing within their ability to capture and detach knowledge from its context without losing its essence. The document provides a mode of transporting abstract knowledge across settings. An opposing discourse argues that formal and abstract knowledge captured in documents represents an impoverished version of the richness of the empirical world and situated knowledge. Abstract models cannot but delete the details of the heterogeneous work that they represent. This creates inflexible systems that will inevitably result in improper functioning when the information system is implemented (ibid.: 405). The first could represent the dream of the universal patient-centered record; the second would find support among many healthcare providers who distrust the viability and timely implementation of large-scale medical information systems.

These positions seem too entrenched; the fundations too essentialist. Moreover, they do not help us explain Marc and Elisabeth's case. These two doctors clearly share practices and work contexts, why don't they also share information system? The question becomes, what roles do the HOSO and Senior Note systems play in their daily work and knowledge sharing practices?

In this paper I attempt to articulate an approach to information technology in organizations that addresses these empirical questions by overcoming the overarching dichotomy between the abstract and situated. I will do so by approaching medical documents as communicative genres. Following the lead from the linguistic anthropologist William Hanks (1990, 1996, 2000), I adopt a view of communication that ties it to practice. Hanks' framework allows us to stay clear of the abstract-transferable versus situated/context-bound dichotomy by studying how knowledge is carried, not by our cognitive processes, but in the way we use language in practice. Documents are no longer mere vessels for abstract knowledge but tools utilized in our unfolding communication, coordination, and knowledge exchanges.

The following section introduces Hanks' analytical approach to communicative practices or what, in the medical context, you could consider documenting practices. This will set the stage for our return to Marc and Elisabeth's case, allow us to analyze their specific documenting practices and help us understand why they deem it necessary to maintain separate on-line note systems.

\section{Genres and the indexical centering of documenting practices}

In linguistics we find a dichotomy comparable to the distinction between abstract and formal knowledge versus the local and situated knowledge divide found in organization and information system studies. One body of linguistic theories focuses on the patterned, abstractable, universal, repeatable, and arbitrary aspects of language and communication. From such a formal perspective, medical communication genres consist of regular groupings of thematic, stylistic, and compositional elements (Hanks, 2000). Generic types of medical documents are defined by differences in features or configurations, no matter the social values associated with them in a given context or the historical conditions under which they come to exist.

A family of approaches promotes the inverse thesis - that our communication is variable, locally adapted, saturated by context, and constantly adjusting to the world beyond its limits (Hanks, 1996). Here, medical genres can be defined as the historically specific conventions that doctors and nurses apply when composing documents and audiences receive them. From this perspective, genres consist of orienting frameworks, interpretive procedures and sets of expectations that are not part of the formal structure (Hanks, 2000), hence the 
ways in which doctors relate to and use medical language define a genre. This approach has a long history in the social sciences where the interaction among language, culture and individual lives is placed at the center of analysis. It fits with Wittgenstein's later writings and phenomenologists such as Merleau-Ponty for whom actions in the world were formative of and not dependent upon the formal structures summarized as grammar.

But, how do we move beyond this dichotomy? Hanks offers one attempt to move beyond the dichotomy between purely formal and socially situated approaches to communication and genre analysis by incorporating formal features of language, while still locating them in relation to everyday and historically specific practices. He does so by shifting our focus away from the content of our communication as the thematized object. Instead, he approaches communicative content as mere resources through which other parts of the world are brought into focus by calling attention to a set of linguistic terms known as "indexicals". In language, such signs encompass demonstratives, pronouns, and other deictics ${ }^{1}$ or "shifters" that relate utterances to their speakers, addressees, actual referents, place and time of occurrence (Hanks, 1996). For instance, a doctor in the emergency room asks a nurse at the nursing station where he can find Mr. Jones. The nurse responds: "Down there." The nurse's utterance "down there" indexes her current location in the nursing station as a ground or center from which she makes reference to the patient laying on a gurney down the hallway. This "indexical centering" embedded in the nurse's answer is a primary part of the physician's interpret-

1 Deictics are words showing or pointing out directly the one referred to and distinguishing it from others of the same class. For instance, the demonstrative pronouns this, that, and those have a deictic function (Encyclopedia Britannica Online, 2001). ation of her utterance because it connects the semantic code with the concrete circumstances of its use. The doctor would have been left rather perplexed had he received the answer "down there" from a disembodied voice over the emergency room's intercom.

The notion of "indexical centering" plays a key role in Hanks' framework. The concept allows us to describe how people routinely make references to places, objects and times that define the relations among the interacting parties. One could argue that the nurse defines the context for her communication with the doctor through the indexical centering of her utterance. In this way, the nurse simultaneously makes reference to and articulates with the context in which she performs her reference.

The nurse, however, is not limited to making references to and articulating with her present context; she could also do this across multiple contexts or places. Imagine that the nurse knows that this newly minted physician started working in the emergency room only yesterday and wants to help the physician understand the organization of the emergency room. She may say: "We keep the orthopedic patients down at the end of the hallway by the elevator. That makes it easy to get them up to the radiology department." In this way the nurse centers her communication on the relation between the emergency room and the radiology department upstairs.

In a similar fashion people can build into their indexical centering references to not only other places but also other times or relations across situations. This point becomes particularly important when we introduce documents as expressive mediums, part of the form through which practices are realized and communication accomplished. Written communication builds around indexical elements to the same degree as face-to-face communication. People may use a document to index their communication within one very limited context. 
Likewise, they may use a document to build an indexical field that points to relations across multiple situations, times, and places.

In Hanks' framework, knowledge exchanges are no longer either situated and context bound or abstract and transportable. People situate their communication in an extremely narrow context by constricting the temporal and spatial range of their indexical field, or they can situate it in relation to times and places far beyond the reach of face-to-face communication. This makes Hanks' notion of "indexical centering" a promising candidate for overcoming the dichotomy between the abstract transportable knowledge versus situated and context bound knowledge. Furthermore, Hanks' framework becomes the key to a deeper understanding of medical documents and the role they play in the communication, collaboration, and coordination among doctors and nurses. The structure of indexical referential terms embedded in different medical document genres can serve as a window into how doctors and nurses position their communication and knowledge exchanges in the complex healthcare field involving countless participants, places and temporal rhythms.

\section{Methodology and field site}

In this article, I attempt to analyze the indexical centering of two medical genres, the interns' HOSO and the senior residents' Senior Note. The analysis falls along two main dimensions in the indexical centering of deictic references: the relationship between the interacting parties on the one hand and the relation between the interacting parties and the object of reference on the other hand (Hanks, 1996: 182).

First, the degree of access between the interacting parties plays an important role defining the indexical centering of particular documenting practices and the text-to-context relations. People's access can vary in degrees of mutual perceptibility or prior knowledge. The interacting parties may have face-to-face interactions with one another or their relationship may be defined by great distance. They may share a common knowledge and full set of referents based on prior experience together or they may never have met. All those factors, according to Hanks (1996), affect the use of deictics and the indexical centering of particular communicative practices.

Second, the relationship between the interacting parties and the object of referent, whether a patient, an object, a place, or a temporal rhythm, can vary greatly. The relationship to the referent may be characterized by a common knowledge or a more or less asymmetric access. Both parties may interact with the referent, e.g. a patient, on a daily basis, or one doctor could be reporting on his or her relation to the patient to another physician who has no prior knowledge of that patient. These aspects of the situation help define the indexical centering of particular communicative genres and the structure of individual deictics and the way they map the interactive space.

To compare and contrast the HOSO and Senior Notes along these two main dimensions of the indexical centering of communicative genres, I look for differences and commonalities in the text-to-context relations across these two genres. More specifically, I focus on the references to author, addressees, and other participants; references to places, place-names, locative descriptions, dates, signatures, spatial and temporal deictics; and other spatial and temporal markers. To protect the privacy of both healthcare providers and patients I have changed all names, dates, institutional identifiers (e.g., record numbers, phone numbers, department names, and institutional names), and sometimes the gender of my informants. The examples of records included in Figure 2 and 3 below are excerpts from field notes that did not contain any patient, clinician, or institutional identifiers. Those identifiers were 
never copied from the originals in the process of the fieldwork.

I draw my empirical case from a 15month, multi-sited ethnographic study in several pediatric healthcare settings, following patients from primary care clinics to emergency rooms and in-patient units in a US metropolitan area. In this larger study I focused on the collaboration among doctors, nurses, and clerical workers, specifically the practices that go into documenting patients' care (Østerlund, 2002). The doctors and nurses were the actors of that study. They cared for patients that moved through the locales they inhabited.

The present paper can be distinguished from the larger study in at least three ways. First, where the larger study positions itself in a broader debate on the social and organizational implications of medical information systems (see (Berg et al, 2003) the present paper takes a far more narrow approach. Here, I hope to highlight the potential of linguistic analysis for sociotechnical studies of medical documents. Specifically, I want to draw attention to Hanks' approach to language as a communicative practice. Analyzing language as practice allows us to tie language use to broader social practices and how document use allows people to deal with the distributed nature of their daily lives and work practices. Secondly, the broader study focuses on the documenting practices of nurses, physicians, and clerical workers. To look across occupational groups and involve both patients' and clinicians' perspectives are important if we want to understand the implications of medical information systems. I do not attempt to address these broader issues in this paper but simply focus on a narrow slice of two physicians' documenting practices. Third, I find it important to take an inclusive perspective on medical information systems and include all records in my analysis, whether made on various note cards, preprinted forms, on-line record systems, and whiteboards. In this work I focus on two record entries in an attempt to illustrate the analytical power of a specific type of linguistic analysis.

Finally, a note on terminology. I prefer to use the term "documenting practices" in place of Hanks' "communicative practices" (Hanks, 1996). Hanks builds his conceptual framework on detailed ethnographic studies of face-toface communication in Maya on the Yucatan peninsular of Mexico or historical analysis of colonial texts from Yucatan. Thus, he does not study the role of documents as an integrative part of peoples evolving communicative practices. By using the term "documenting practices" I hope to highlight the central role documents play in the structuring of everyday work practices and knowledge sharing in organization. In short, my unit of analysis is the documenting practices of doctors and specifically the practices that go into documenting patients' care.

The argument is structured as follows: Before we turn to the two dimensions of indexical centering outlined above I will briefly look at the more formal genre features that go across these two documents. In other words, we will start out with a more "content-based" analysis focusing on formal features characterizing these two texts and medical documents in the US in general. This is first followed by a discussion of the relationship between the interacting parties, and secondly an analysis of their relationship to the referent, that is, the patient.

\section{Content-based Analysis: SOAP}

Elisabeth's senior notes (Figure 2) and Marc's HOSO (Figure 3) both adhere to the same general genre format: the subjective data, objective data, assessment, and plan (SOAP). Figure 1 summarizes the issues subsumed by this acronym. Nurses and doctors engage this narra- 
Figure 1-SOAP: Subjective Objective

Assessment and Plan

Subjective Data
Identifying Information
Chief Complaint (CC)
History of Present Illness (HPI)
Past Medical History (PMH)
Medication and allergies
Family History (FH)
Social History + habits (SH)

\section{Objective Data}

Review of Systems (ROS) (including an ordered list of every relevant organ, noting the present or absent symptoms referable to that organ)

Physical Exam (PE)

Labs

X-ray

Special tests

\section{Assessment}

Diagnosis or differential (list of possible diagnoses)

\section{Plan}

Treatment regiment or other action taken by doctor or nurse

tive structure when presenting individual and patient histories both verbally and in writing. Many medical schools and teaching hospitals strongly promote this organizing structure for history giving, including Kiltham Hospital. Marc and Elisabeth readily recite the SOAP elements if asked.

The SOAP builds on a widespread genre for medical histories in the US. Structured formats for history records can be found as early as the nineteenth century (Epstein, 1995). The present system began to be established in the early nineteenth century and became codified in the last decade of the century. In the nineteen sixties the American physician Lawrence Weed introduced the SOAP format as the guiding structure for his "problem-oriented patient record" in an attempt to design and de- pict clinical work as a type of experimental or "scientific" activity. Weed explicitly labeled the distinct steps in the clinical process (i.e. SOAP) as elements of the scientific method. The hope was to lay open medical practice to scientific analysis in a new and thorough way. The individual steps of the experiment, the definition of the starting point, the planning of the intervention, and the observation of the outcome should be discerned and judged. Through the problem-oriented record, the doctor "is able to organize the problems of each patient in a way that enables him to deal with them systematically" (Berg, 1997b: 23; Weed, 1968). For a thorough discussion of Weed's writings and their attempts to standardize medical work see Berg (1997b) and Timmermans \& Berg (2003).

The patient histories found in Elisabeth's Senior Notes follow the SOAP format nearly to the letter. The header and the first paragraph of the two histories included in Figure 2 summarize Dylan Jones and Anna Hague's "subjective data". These include their name, record number, an acronym, ASSN, which means that their case is assigned to an attending physician in the hospital and not their own primary care doctor; this is followed by, admission date, the name of the intern in charge of their case, and the patient's age, and chief complaints. Chief complaints can be symptoms or diagnosis or a mix of the two. We learn that both patients, only a few weeks old, suffer from viral bronchiolitis. In addition Dylan Jones has a newly diagnosed heart defect (i.e. ASD), possible gasteroesophageal reflux (GERD), and failure to thrive (FTT). The latter is a catchall diagnosis for children who do not follow normal growth patterns. The first paragraph summarizes in a telegraphic style "history of present illness", "past medical history", "medication and allergies", "family history", and "social history". Dylan presented in the Emergency Room (ER) after three days of coughing, vomiting, and decreased eating. The ER doctors admitted him to 
Figure 2 - Excerpt from Senior Notes Showing First Page and Two Entries

Notice: This figure contains no real patient, clinician, or institutional identifiers.

PEDIATRIC TEAM B

Wednesday, February 23, 2002

\begin{tabular}{|c|c|c|c|}
\hline Senior Residents & Interns & Medical Students & Attendings \\
\hline Elisabeth Lave \#124 & Marc Bergger \#343 & Heinrich Schreiber \#89 & Patrick Dreier, M.D. (ASSN) \#482 \\
\hline Oscar Hanks \#1193 & Donna Ito \#129 & John Van Fennen \#87 & Tina Law, M.D. (Teaching) \#104 \\
\hline Pei Lin \#1268 & Gabriel Callon \#432 & Chingning Lo \#43 & \\
\hline Roger Moore \#1596 & Jennifer Latour \#987 & Jonghoon Kim \#34 & \\
\hline
\end{tabular}

$\begin{array}{lrlll}\text { Daily } & \text { 7:30 am } & \text { Work Rounds } & \text { 10E Conference Room } & 253-4931 \\ & 9: 30 \mathrm{am} & \text { Radiology Rounds } & \text { 10E Ward } & 253-8931 \\ & 10: 00 \mathrm{am} & \text { Senior Rounds } & \text { 10E Fax } & 253-9318 \\ \text { Tuesday } & 12: 00 \mathrm{pm} & \text { Team Rounds } & \text { PTB Senior Call Room } & \text { E53-598 } \\ \text { Friday } & 12: 00 \mathrm{pm} & \text { Moe Conference } & & \end{array}$

$\begin{array}{llllll}10 \mathrm{E} & \text { Jones, Dylan } & 123 & \text { ASSN } & \text { Marc } & 1 \text { mo RSV bronchiolitis, ASD, PPS } \\ 10 \mathrm{E} & \text { Carlile, Jim } & 667 & \text { PHA } & \text { Donna } & 12 \text { do UTI, persistent fever, leukocytosis } \\ 10 \mathrm{E} & \text { McGill, Dede } & 564 & \text { HPHC } & \text { Donna } & 3 \text { yo cervical adenitis } \\ 10 \mathrm{E} & \text { Arc, Noah } & 251 & \text { PHA } & \text { Marc } & 11 \text { month fever, tachypnea,? acidosis } \\ 10 \mathrm{E} & \text { Finnen, Maria } & 759 & \text { ASSN } & \text { Marc } & 5 \text { wo RSV+ bronchiolitis, ICU transfer } \\ 10 \mathrm{E} & \text { Bush, George } & 228 & \text { PHA } & \text { Oscar } & 5 \text { mo RSV+ bronchiolitis, ICU transfer } \\ 10 \mathrm{E} & \text { Panama, Anna } & 126 & \text { PHA } & \text { Marc } & 2 \text { month old vomiting/cough, hx of FTT } \\ 10 \mathrm{E} & \text { Hague, Anna } & 846 & \text { ASSN } & \text { Marc } & 5 \text { wo RSV + bronchiolitis, ICU transfer. } \\ 10 \mathrm{~W} & \text { Tyre, Marcy } & 352 & \text { HVMA } & \text { Oscar } & 4 \text { wk mild bronchiolitis, murmur, social } \\ 10 \mathrm{~W} & \text { Willey, Vienna } & 998 & \text { IMMUNO } & \text { Oscar } & 8 \text { yo ataxia telangletasia, pulmonary AVM } \\ 11 \mathrm{E} & \text { Yate, Deborah } & 674 & \text { ARMS } & \text { Donna } & 9 \text { do conjunctivitis, r/o sepsis } \\ 11 \mathrm{E} & \text { Kim, Jooh } & 375 & \text { ASSN? } & \text { Marc } & 6 \text { mo bronchiolitis } \\ 11 \mathrm{E} & \text { Johnson, Lotte } & 242 & \text { ASSN } & \text { Donna } & 7 \text { do r/o sepsis } \\ 11 \mathrm{E} & \text { Deed, Graham } & 442 & \text { ASSN } & \text { Jen } & 3 \text { yo RML pneumonia, first RADexacerbation } \\ 11 \mathrm{E} & \text { Mogadi, Chenge } & 889 & \text { PHA } & \text { Jen } & 9 \text { y/o HSV vaginitis } \\ 12 \mathrm{~S} & \text { McDonald, Mike } & 764 & \text { RHEUM } & \text { Oscar } & 15 \text { yo SLE, worsening BUN, left foot pain } \\ 12 \mathrm{~S} & \text { Cetina, Virginia } & 372 & \text { ASSN } & \text { Donna } & 10 \text { yo viral meningitis } \\ 12 \mathrm{~S} & \text { Potter, Forrest } & 115 & \text { ASSN } & \text { Marc } & 11 \text { wk old with Salmonella bacteremia }\end{array}$




\begin{tabular}{llllll}
$12 \mathrm{~S}$ & Georgia, Natalia & 151 & RHEUM & Donna & 15 yo MCTD, LLL pneumonia \\
$12 \mathrm{~S}$ & Penn, Sean & 785 & HEME & Jen & 14 yo Hgb SS, VOC (necklabd pain) \\
$12 \mathrm{~S}$ & Li, Jean & 874 & HEME & Marc & 7 yo Hgb SS, abdominal VOC, s/p ICU \\
$12 \mathrm{~S}$ & Fisher, Hugh & 659 & ASSN & Marc & 9 mo Trauma X, shaken-baby syndrome \\
PB & Annaby, Sheena & 097 & HEME & Jen & 15 yo Hgb SS, abdominal VOC, NO study \\
& & \multicolumn{4}{c}{$\sim \sim \sim \mathbf{1 0}$ E $\sim \sim \sim$}
\end{tabular}

Jones, Dylan $123 \quad$ ASSN $\quad 2 / 16 \quad$ Marc 1 mo RSV bronchiolitis, ASD, PPS

1 mos old presented with cough $\mathrm{x} 3$ days, question of decreased PO and vomiting. Got r/o sepsis for fever in ER. Recently admitted $2 / 7$ for rule-out sepsis. In ER, taking pedialyte PO, 37.6, 172, 48-88, 100\%. Not wheezing, no G/F/R. CXR with RML atelectasis. WBC=11.4 (28P,55L,4Bd), Hct=31.5, Plt=455. Bicarb 18. UA neg. Lytes wnl. Urine and blood cultures pending. Mom and child live in a shelter. PMH Born FT 7lbs 5 oz. On 01-15, reportedly $8 \mathrm{lbs} 12 \mathrm{oz}$. On admission 7 lb 14 oz.?FTT

RESP: increased interstitial markings prob due to pulm edema, now resolved;?patch infiltrates c/w Chlamydia; vapo nebs prn. Initially thought the tachypnea was due to CHF. Gave Lasix. On 2/18, had RR to 110. Gave Alb and Vaponebs with out improvement. ABG showed 7.45/24.9/127/17. CXR showed hyperinf SSA. Transferred to ICU. Tachypnea improved. Respond to Vaponebs but not albuterol. On RA with good sats. RSV came back Positive!

CV: CXR with heart size upper limits nl, 4Ext BPs nl, R sided axis on EKG. Liver edge down, ECHO with large ASD, and left PPS and RV hypertension. On floor, tried to diurese with lasix. Now stopped. Cardio following - now things resp issues not cardiac. F/u in clinic for ASD.

FEN: newborn screen wnl; came in only $3.6 \mathrm{~kg}$. Lost $0.8 \mathrm{~kg}$ after diuresis. Looks cachetic with decreased muscle bulk.?poor nutrition,. W/U for FTT. They placed an NJ tube in ICU due to resp distress and FTT issues. Started Prosobee at $5 \mathrm{cc} / \mathrm{hr} /$ (hx of rash with Enfamil). Nutrition consult. Also? GERD due to hx of back arching - started Zantac. Increased to full feeds on floor. NJT pulled and now po feeding, gaining weight.

ID: cultures pending; started on erm for?atypical - changed to Azithro in ICU x 5 days (ends 2/24); rsv positive.

SOCIAL: 4438700 x987 Peter NP. Mother lives in a shelter. 2 step-children SW involved.

Hague, Anna $846 \quad$ ASSN 2/15 Marc 5 wo RSV + bronchiolitis, ICU transfer.

5 week old FT/LGA previously healthy with RSV + bronchiolitis transported from Common Hospital $1 / 29$, in ICU intubated $1 / 29$ to $2 / 12$ (on Hifi for portion), transferred to floor 2/15.

Pulm: Wean $\mathrm{O} 2$ prn. Pulm consulted regarding weaning of diuretics. Attempted to $\mathrm{d} / \mathrm{c}$ but developed fluid overload requiring Lasix $1 \mathrm{mg} / \mathrm{kg}$ so restarted. Now on room air.

$\mathrm{CV}: \mathrm{H} / \mathrm{o}$ murmur. Echo showed PPS. Currently stable.

ID: RSV+. Trach cultures grew S. aureus (sensitive to oxac \& clinda), S pneumoniae, and Morazella. On Zosyn and Vanco in ICU initially, changed to Unasyn and Ampicllin, d/c 2/11. Now afebrile off antibiotics, Eye d.c PSA and serratia. Gentamicin \& Ilotycin eye ointment.

GI: On NJ continuous feeds when transferred from ICU. Now on po feeds.

FEN: In ICU, high HC03 (40's) due to lasix. Chlorothiazide \& spironolactone PNJT q 12 hrs, follow lytes qD. Bicarbs down to 30s. May need to go up on diuretics b/c UOP not great.Heme: Hct 29.

Neuro: On methadone and ativan taper. Low NAS scores so d/c'd 2/17. Increased sweaty and irritable on 2/19, NAS score 11 - given small dose of Ativan.

Dispo: Discharge pending when off 02, full feeds, and sedatives weaned. 
Figure 3 - Excerpt from HOSO Showing Two Entries

Notice: This figure contains no real patient, clinician, or institutional identifiers.

KILTHAM HOSPITAL

HOUSE OFFICER SIGNOUT

Wednesday, February 23, 2002 06:56:12

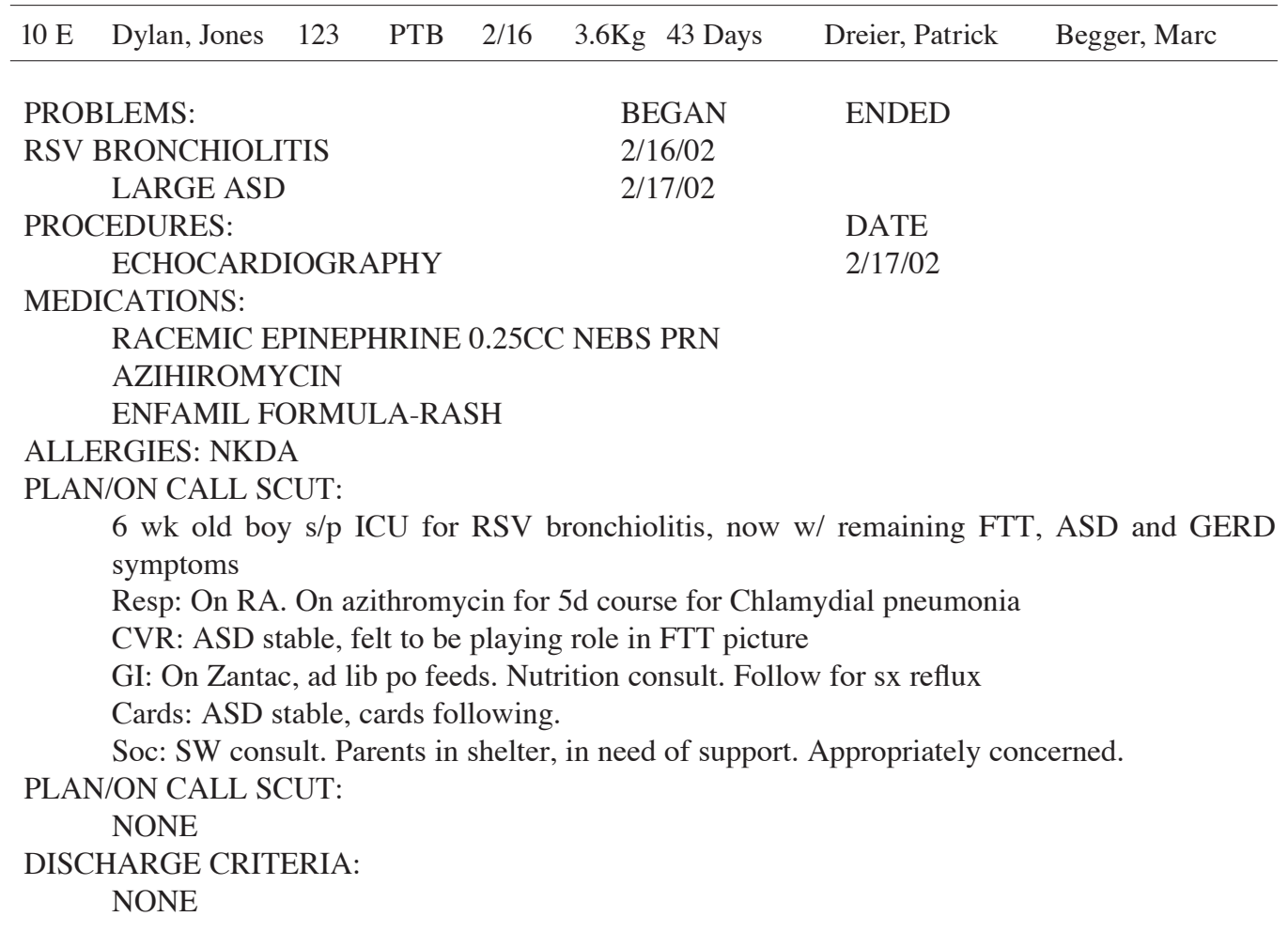

\begin{tabular}{|c|c|c|c|c|c|c|c|c|}
\hline $10 \mathrm{E}$ & Hauge, Anna & 846 & PT & $1 / 28$ & $5.6 \mathrm{Kg}$ & $2 \mathrm{Mos}$ & Dreier, Patrick & Begger, Marc \\
\hline
\end{tabular}

$\begin{array}{lll}\text { PROBLEMS: } & \text { BEGAN } & \text { ENDED } \\ \text { RESP DISTRESS } & & 2 / 23 / 02 \\ \text { RSV BRONCHIOLITIS } & 2 / 23 / 02 \\ \text { PROCEDURES: } & \text { DATE }\end{array}$

NONE

MEDICATIONS:

ALBUTEROL PRN

TYLENOR PRN

ALLERGIES:

NKDA

PLAN/ON CALL SCUT:

NONE

DISCHARGE CRITERIA:

NONE 
rule-out infection as the underlying course of his symptoms. The next few lines summarize his vital signs taken in the ER and the procedures he went through. Finally, we learn that he lives with his mother in a shelter and that he gained weight in the first weeks after birth, which he subsequently lost again. In Anna's history we learn that she was born large and healthy at full term. Soon after, she got viral bronchiolitis leading to her first admission at a small suburban hospital (Common Hospital). They transferred her to the ICU at Kiltham hospital where she was intubated for two weeks before getting transferred to one of the hospital's pediatric medical units, 10 East.

The rest of Dylan's and Anna's histories review what is considered relevant organ groups for their cases, or rather relevant sub-specialties in the hospital. In Dylan's case this includes respiratory (RESP), cardiovascular (CV), fluid electrolyte nutrition (FEN), infectious Disease (ID), and social services. In Anna's case the note calls attention to pulmonary (Pulm) cardiovascular, infectious diseases (ID), gastrointestinal (GI), fluid electrolyte nutrition, and neurology. Each of these subsections reviews Dylan's and Anna's "objective data", "assessment", and "plan".

In addition, the authors elaborate "history of present illness" as they sum up test results and give their assessment and plan. In other words, the review of each organ group contains a small narrative that justifies the actions, assessment and plans taken. For instance, in regard to Dylan's cardiovascular system (CV) we learn that an X-ray showed an enlarged heart and an echogram later unveiled a heart defect. Treatment with lasix was tried but later abandoned. The cardiovascular team now follows Dylan's case, and they do not perceive his heart problems as directly related to the respiratory problems triggering his hospital admission. Based on these analyses the plan is to pass over the responsibility of Dylan's long-term cardiovascular care to the outpatient clinic.

In Anna's case, we learn under the pulmonary subheading, for instance, how she received concentrated oxygen through a mask. This was later discontinued and she is currently breathing room air. An oxygen mask may still be used if found necessary. After an attempt to wean her of diuretics failed, Anna developed fluid overload - leading to the involvement of the pulmonary team as consultants. Anna's history concludes with a disposition/plan: she will be discharged as soon as she does not require an oxygen mask, eats normally, and has been weaned off the sedatives originally started in the ICU as part of an aggressive treatment regiment. Dylan's history does not contain a separate section on his disposition, which could mean that the physicians have not yet made a discharge plan for him, as too many questions remain unanswered.

Marc's HOSO (Figure 3) stands out as a significantly more schematic and truncated summary of Dylan and Anna's cases compared to Elisabeth's Senior Note. For instance, the HOSO reduces Anna's case to a few lines. We learn that the physicians consider her respiratory distress and viral bronchiolitis as treated by today's date. In addition they can give her the medications Albuterol and Tylenol if needed. Nevertheless, the HOSO contains most of the SOAP genre elements - though, presented out of order. The HOSO sums up Dylan and Anna's "subjective data" in the header, and under the sections on problems, procedures, medications, allergies, and the first line of the section "plan/on call scut". The term "on call scut" refers to the work pending for the intern in charge. In Dylan's case we learn that his bronchiolitis and heart problems were diagnosed on October $6^{\text {th }}$ and 7 th. On the same day the echocardiography was conducted. Equally important to the interns responsible for the patients' medication, the HOSO contains a list of the medications currently given to Dylan. 
One sentence captures the history of his current illness; originally he was admitted to the ICU for bronchiolitis. Since then three other issues have emerged including failure to thrive, heart problems and reflux.

Comparable to the Senior Notes the HOSO envelops the "objective data", "assessment", and "plan" into a review of systems compiled in the section, plan/on call scut. The HOSO does not review test results but simply highlights important issues and points to the subspecialties involved. In Dylan's case we learn, first, that he is on room air and on a five-day course of medication for pneumonia. Second, the cardiac consulting team follows Dylan's heart problems, which they believe may be the underlying cause to his failure to thrive. Third, the nutrition consult team follows Dylan's reflux symptoms and has put him on a special baby formula diet (i.e., Zantac). Finally, the HOSO highlights Dylan and his family's living situation and that a social worker team follows his case.

In short, the Senior Notes and the HOSO include the same formal genre elements. With small variations the two records follow the SOAP format. What stands out, are the significant differences in length and detail across the two record types. The senior note provides a comprehensive account of Dylan and Anna's care. The HOSO is conspicuously brief in comparison; Anna's case seems astonishingly abbreviated. The HOSO leaves us no sense of her treacherous tour through the healthcare system starting at one hospital, transferred to Kiltham's ICU and later moved to an inpatient unit, 10 East, where she has been treated with methadone for withdrawal symptoms caused by the intensive medication she received in the ICU. These significant differences in length and comprehensiveness across the two records raise the questions: why these differences? More specifically, why do busy interns like Marc spending more than 90 hours a week in the hospital take the time to write the HOSO if he could just read the senior resident's more complete account of his patients' histories? Or, the senior resident could have the interns write a more detailed note freeing up time for the senior resident to engage in research or other high prestige activities? In an attempt to address these questions we will now turn to an analysis of the indexical centering of the HOSO and Senior Notes respectively.

\section{Indexical Centering}

A key element in our practice-based analysis of medical records is the grounding of the more generic genre elements in their indexical context. We recall that indexicality is a semiotic mode in which signs stand for objects through a relation of actual contiguity with them (Hanks, 2000: 151). Pronouns, demonstratives, and other "shifters" relate utterances to their speakers, addressees, actual referents, places and times of occurrence. Indexical centering plays a principal role in the interpretations of medical documents as it connects the evaluative and semantic code with the concrete circumstances of its use. The Senior Notes and HOSO genres embody specific kinds of public address by a collective of speakers, before a collective of addressees and about a group of patients and colleagues, all located in a carefully constructed "here" and "now". First, I analyze the indexical centering of the relationship between the interacting parties. Second, I turn to the deictic system defining the indexical ground of the relationship between the interacting parties and their object of reference, the patient.

\section{Interacting parties: Addressivity, spatial field, and temporal field}

The indexical centering of the relation between the interacting parties can be broken down to the deictic references to participants, or "addressivity," and the spatial and temporal 
fields for interaction. Let us start out looking at what Bakhtin calls a genre's "addressivity" (Bakhtin, 1986). Different genres correspond to distinct conceptions of the addressers and addressees. The addresser or addressee may be an individual, a social group, contemporaries, successors, an unconcretized Other, or a combination (Hanks, 2000: 151).

\section{Addressivity and participants}

In the Senior Notes and HOSO we do not find any explicit address apart from the name of each document genre. Senior Notes address senior residents and House Officer Sign Outs (HOSO) address house officers, the latter being physicians in Kiltham's residence programs, including interns (first year residence), second and third year residence. In Kiltham interns predominantly use the HOSO. Equally important and in contrast to the majority of medical documents, neither the Senior Notes nor the HOSO identify the speaker. We find no signatures or specification of who tailored these documents. In the HOSO we do find Marc's name in the header to Dylan and Anna's entries as the "Intern". This does not mean that Marc is the sole author of the record; simply that he is in charge of these two patients during his rotation in the Pediatric Team B.

Senior Notes and the HOSO are communal documents where a distinct social group constitutes each genre's collective addresser and addressee. Most likely three or four senior residents have been involved in the writing of Dylan and Anna's histories. Likewise, Marc did not write all parts of the HOSO. For instance, if we return to the day where Marc and Elisabeth examine Dylan and Anna, we find that in the late afternoon, just before going home, Marc signs-out his patients to one of his fellow interns, Donna, who is staying in the hospital overnight. They use the HOSO to structure their conversation. Overnight, Donna uses the HOSO to structure her activities. And if anything happens to Dylan or Anna, she will add the event to the HOSO. Elisabeth shares her notes in the Senior Notes on-line system with other seniors only.

On her on-call nights Elisabeth covers for not only patients at Pediatric Team B but also two other departments. When the senior residents in those other units sign-out, Elisabeth prints out a new version of the Senior Note containing all patients currently in all these three units. The Senior Note printout can easily contain $30-40$ patients. In other words, Elisabeth builds on other senior residents' entries rather than writing Dylan and Anna's histories anew. Senior residents in the ICU most likely wrote parts of these two histories; Elisabeth and other senior residents later edit those earlier entries to make them reflect the current status of a patient. When I first started my field research, these practices puzzled me a great deal. One late afternoon I asked a senior resident why he just spent 45 minutes editing entries originally initiated by other senior residents. He responded:

"I'm anal. I want the notes to follow a specific setup. No empty spaces. Look at this one [pointing to a particular voluminous patient entry on the screen]. It's so long that you think that it's a complicated case, but it's just a 4 month old with bronchiolitis."

In short, Senior Notes and the HOSO stand out as communal documents where authors and addressees overlap and individual contributors take on the role of contemporaries and successors interchangeably. People spend hours making factual changes but also minute modifications to the records' lengths and style - thereby adhering to communal genre requirements about how best to signal, for instance, the potential workload involved in each case.

Where the HOSO and Senior Notes contain no explicit speaker and address, they do contain references to the current community of participants or contemporaries. At the beginning of the Senior Note we find a table listing the 
names of senior residents, interns, medical students, and attendings. The four senior residents named on the left hand side are all contemporaries to the current record and this group of patients. Elisabeth's name goes first signaling that she is currently in charge of the patients admitted to Pediatric Team B. The other three senior residents all cover for her on different nights of the week. In the HOSO we would get a comparable sense of the author/addressee contemporaries if we printed out the entire HOSO for Pediatric Team B. If we read the right hand side of the headers for each patient, we would find the names of the four interns on Marc's team. Each of them would be assigned as responsible for a portion of those patients.

Apart from the names of senior residents and interns involved in their respective communal system of "addressivity", we find a host of names referring to other participants. These include names of medical students, attending physicians, patients, acronyms for various medical services (e.g. Cardiac, Heme, etc.) and other professional groups (e.g., social workers). In contrast to the implicit composition of speakers and addressees among the interns and senior residents, we find an explicit structure referring to other collaborators, their relationships and interdependencies.

Starting with the Senior Notes, the top of the document includes a four column table listing, not only the senior residents producing and using the senior notes, but also the interns, medical students and attending physicians with whom they currently collaborate on Pediatric Team B. The table demarcates a group of contemporaries to the present document. The sequence of the four columns hints at the power relations among the four groups. The senior residents oversee the work of the interns, who manage and mentor the medical students. The attending physicians watch over the entire team by taking on a supervisory role. One attending physician is responsible for the patients not attended to by their pri- vate physician or other sub-specialties, in this case Patrick; the other, Law, supervised the teaching of the medical students. Given that the attending physicians hold the ultimate responsibility for patient care, one may expect to find them in the first column. However, the senior residents" "ownership" of the record most likely explains this inconsistency in the sequencing. In short, the table recaps the interacting parties. It goes beyond the relations among speakers and addressees by including the interacting parties involved in the care for a group of patients.

\section{Spatial field}

Elisabeth's Senior Notes contain an explicit structure demarcating the spatial dimensions for her collaboration with the other members of Pediatric Team B. Following the table we find on the right hand side a list of three important places and their phone number: 1) Pediatric Team B uses the 10 East Conference Room as their base for writing records, hanging out and working rounds. 2) The conference room is located on the 10 East Ward next to the nursing station where all calls to the ward get directed. 3) The PTB Senior Call Room is where senior residents hope to catch a few hours of sleep when they are on-call at night.

We also find a number of less explicit spatial markers embedded in the first section of the Senior Note. First, notice the pager numbers follow the physicians' and medical students' names in the first table. One can consider these pager numbers a spatial reference to mobile individuals or what Mizuko Ito calls "networked localities" (Ito, 1999, 2001). Building on the idea of networked locales one could also read the patients' record numbers in the third column as spatial references. Physicians often find that a patient's record number is a more reliable locator than their name, the latter often being misspelled or the same name held by several patients. Second, we find a blurring of the distinction between place and partici- 
pants in the fourth column in the senior note's table of content. This column summarizes the service in charge of each patient. PHA is the hospital's outpatient clinic and a physical place. In contrast, IMMUNO stands for immunology. This sub-specialty does not have its own clinic per se where patients go. The immunology team moves from ward to ward to consult on specific patients. Much like the pager numbers these names refer to specific social spaces and participants, which may and may not be associated with a physical place. Interns and senior residents pay attention to those spatial signals afforded by various technologies such as their pagers. Over lunch one day a group of interns discussed how best to discern the physical location of a page based on the call back number displaced. The team uniformly agreed that when a page was coming from the hospital's cafeteria, they expected it to be cardiac team member trying to get in touch with them. The cardiac team tended to gather and work in one corner of the cafeteria close to the library.

\section{Temporal field}

Temporally, interns and senior residents structure their HOSO and Senior Notes use around change of shift, rounds, and sign-out. The first thing an intern or senior resident does when arriving at work, is to log-on to the HOSO or Senior Notes respectively. In doing so they determine if their on-call colleagues added any significant information over night. During the day, and in particular in the afternoon and late evenings, interns and senior residents update their communal note systems. In the afternoon there is a flurry of activity in the 10 East conference room when interns get ready to sign-out their patients to the on-call person. When two interns were asked what time of day they considered the most important, they answered in unison: "Sign-out". Interns update the HOSO, and during sign-out use it to structure their report to the on-call colleagues. In their conversation they focus on the patients that may need attention during the night, difficult orders and other tasks.

As in the case of the spatial references, the Senior Note starts out by demarcating an explicit temporal structure for their work in Pediatric Team B. With a glance at the top of the senior note we learn that the daily work for the senior residents structure around working rounds at 7:30, radiology rounds at 9:20 and Senior rounds at 10:00. All the members of Pediatric Team B outlined in the table participate in work rounds and radiology rounds. During those rounds the team will go over each patient case, typically initiated by the intern or a medical student, recounting the patient's history and progress. Radiology rounds take place in the radiology department where the team will huddle around a radiologist who will go over the latest x-rays and scans. Afterwards the group splits up. The senior residents will go to their Senior Rounds while the rest of the team starts working on individual patient cases. In the late afternoon Elisabeth will sign-out to the senior resident staying over night. Marc will sign out to the intern staying in the hospital over night. If they are on call themselves, the other senior residents or interns will sign out their cases to them.

These two communal document genres become an integrated part of the hospital's staggered structure of coverage where staff groups in sequential shifts will overlap with one another for several hours or just 15 minutes. The notes help smooth transitions by providing incoming doctors with immediate sources of information and reference from the moment the outgoing staff members leave the hospital. This explains why house-officers make an extra effort to write particularly detailed notes in the HOSO and Senior Notes on the last day of their rotation. Interns strive to discharge all their patients but if that is not possible, they write to capture as much detail as possible to make it easier for the next intern to take over their patients. 
In summary, interns and senior residents associate specific times of the day with their communal document genres. Each group carefully maintains the coordination among its members in regard to these times and the documenting practices involved. These two communal document types tie closely to two patterns of temporal coordination described by Zerubavel in his study of hospital work, that is, temporal complementarity and staggered coverage (Zerubavel, 1979). The senior note and HOSO allow the senior residents and interns to maintain temporal complementarity, permitting, for instance, Marc to cover for Donna, his fellow intern, when she goes home at night and vice versa. The HOSO plays an important role in supporting staggered coverage. It is exactly in the overlaps between shifts that doctors (and nurses) discuss those communal documents.

At this point, one may ask why the HOSO, compared to the Senior Note, does not contain a comprehensive mapping of the participants, temporal and spatial structures making up its indexical field. The key question here is the degree to which interns have access to interns and senior residents have access to senior residents. We find many graduations of mutual access and the question becomes: to what degree do the interns share mutual perceptibility and prior knowledge about their space of interaction compared to the senior residents? The answer is embedded in their spatial and temporal fields. For the five weeks Marc and his three other interns are on rotation in Pediatric Team B they share collaborators, spatial structure and temporal rhythm. Every morning they listen as they each present old and new patients. At night they cover for each other. In contrast, Elisabeth works within the interaction field of Pediatric Team B during the day, but at night she covers for other teams with different participants, spatial and temporal structures. When on-call at night, Elisabeth prints out a fresh senior note demarcating her new and larger space of interaction. Marc and his fellow interns do not need to be reminded of the spatial and temporal dimension of their interaction field every time they look at their HOSO. It is the same for several weeks and, in case they should forget, they do keep a log of it on a large whiteboard in the 10 East conference room where they typically type up their notes. Elisabeth does not share such a symmetric space with her fellow senior residents.

Senior residents happily spend their breaks discussing the details and pitfalls of their rotation cycle and how it cannot be compared to that of the interns. One evening in the houseofficers" "dungeon", a group of three senior residents and five interns eat their cafeteria dinners. On a large round table one finds remnants of other house-officers' meals taken earlier in the evening and scraps of paperwork left behind. Bags and other personal items lay on available surfaces. An intern plucks random cords on a piano in the corner. Seated around the table, three senior residents discuss a particularly weak point in the way senior residents sign-out (take over from each other) during the weekend. During the weekend the reduced number of senior residents do not have time to go to all the morning rounds, which means that no senior resident will see patients admitted overnight by the night-float (a third year resident on night duty). Sean, one of the seniors, states in a grave voice: "So, there may be some patients who have been here for 24 hours and nobody has seen them or knows what the issues are. It's scary!" The intern at the piano says in a small voice: "But the interns have seen them and know." To which Sean promptly reply: "Oh yes, but we have the code pagers (the pagers called if a patient goes into a coma or experiences a sudden and serious deterioration of health). If you get a code you would like to know who the patient is and what the problem is." Another senior adds: "Yeah, you come up to the floor, who is this? Is it asthma, strep...!? 


\section{The Referent: Dylan and Anna's histories}

It is nearly impossible to talk about the relationship between doctors without also specifying their relations to patients, as the past section illustrates. Relations among the interacting parties constitute only one dimension of the HOSO and Senior Note's indexical ground: the other dimension is their relation to the referent, the patient. ${ }^{2}$ The HOSO and Senior Note operate with two levels of relations between the referent and the interacting parties. The two genres can be read as a compilation of individual patient histories, each specifying the relationship between caregivers and a patient. The HOSO and Senior Notes also present all patients as one object of referent, that is a compilation of all patients currently admitted to Pediatric Team B. This means that Marc and Elisabeth not only read their notes when addressing individual patient issues; they use the records to give them an overview of their current workload, i.e. all the patients admitted to the team. In other words, the object of referent can be see as either an individual patient or part of a cohort.

The bold section following the table and timetable on the first page of the Senior Note illustrates this latter point. This section constitutes a table of contents by compiling all the headings from each patient history in the present version of the Senior Note. Each line summarizes the department, patient, service in charge, intern in charge, the patient's age, and chief complaint or diagnosis. As an entity this table of content outlines a body of work characterized by a particular configuration of participants and places. For instance, in the Senior Notes' table of content each line starts with the name of the ward where a patient is

2 One could argue that patients and/or their relatives serve as not solely "referents" but also interact with the doctors. The patient's role in the healthcare encounter is obviously central but for the purpose of this paper I will maintain my focus on the indexical structures within the two record systems. admitted. We see that the team has eight patients on 10 East, two patients on 10 West, five patients on 11East, and seven patients on 12 South. In other words, the left hand column serves as a flexible map. The team's patients are distributed all over the hospital and the configuration of these locations changes through the day as new patients get admitted and others discharged or transferred from the wards. A boy suffering from Sickle Cell disease is admitted to 12 South, as Elisabeth and Marc write their notes. When Anna gets discharged in a few days the team may get another patient on 11 West, and if Dylan's condition should deteriorate and require a transfer back to the ICU, another patient may take his place on 10 East. Elisabeth refers to her senior note when determining where to go next or where the nurse calling her about where a patient, for example, Hugh Fisher, may be located. In addition, the table specifies the distribution of team members in relation to this larger body of work. With a glance we can tell where Marc's patients are admitted in comparison to Donna's patients. We know how many patients Patrick has been assigned and so forth.

The interns use the HOSO in a similar fashion despite its lack of a summary table. Each new history starts with the patient's location. A glance at the HOSO tells us that Dylan and Anna both are admitted to 10 East. If more histories had been included in Figure 3 one would see that they are sequenced the same way as the Senior Note starting with the wards at the lowest floors and then moving up. Marc and his fellow interns will flip through the HOSO when planning their day or where to call if he needs to know whether the patient's one primary care doctor is responsible for the care or an attending physician in the hospital is assigned to the case. In short, these two document genres serve as flexible maps outlining the ever-changing relation between the physicians on Pediatric Team B and all patients currently admitted to the service. 
With this broader definition of the object of reference in mind let us now turn to the individual patient histories and the indexical field those narratives outline in the interactions between physicians and patients. I start out with the participants, followed by a discussion of the spatial and temporal fields.

\section{Participants}

The HOSO and Senior Note outline another level of participants in the body of Dylan and Anna's histories. These participants do not have enduring relationships with the members of Pediactic Team A. Their interactions are defined by the requirements of individual patients' cases. For instance, Dylan's Senior Note history mentions four groups following his case: "Cardio following", "Nutrition consult", "Peter NP", "SW involved" (i.e., social work). In contrast to the earlier section of the senior note, we find no proper nouns designating particular participants, with the exception of Peter, a nurse practitioner in the shelter where Dylan's mother lives. The same is the case in Anna's history. We learn that the pulmonary team has been consulted (i.e., "Pulm consulted"). The lack of proper names referring to the physicians involved from the different services may be explained partly by the loose relationship between the consulting services and the members of Pediatric Team B. Furthermore, the members of each subspecialty rotate through their teams. The Pulmonary team coming up to 10 East to check on Anna could easily be composed of different individuals from one day to the other. In contrast, the nurse practitioner, Peter has promised to follow up on Dylan's case when he returns to the shelter, an arrangement that has been set up by the nurses on Dylan's unit, 10 East.

The histories stand out by their lack of pronouns, and relatively few descriptive epithets and vocatives referring to participants. A single descriptive epithet refers to Dylan and Anna in the first sentence of their histories, in Dylan's case: "1 mos old" (one month old). The rest of the history mentions neither Dylan's name nor any pronouns referring to him. Each new sentence seems to point back to Dylan's name in the history's header or the descriptive epithet opening the history. Only in the fourth line are the vocatives "mother and child" used in the sentence: "Mother and child live in a shelter." When it comes to healthcare providers we find one pronoun referring to physicians in the entire history: "They placed an NJ tube in ICU... " (line 14). In the rest of the text the vocatives "ICU" and ER seem to point to places and not people. However, the boundary between participants and place names blur. The nouns "ICU, "floor," and "ER" refer to both physical places, but also a collective of healthcare providers. In comparison, the "Pulm" (Pulmonary team in Anna's history) signify a group of healthcare providers not associated with a physical place.

In the HOSO we find a comparable indexical centering of the relation between participants and patients through the use of pronouns, epithets, and vocatives. Dylan is referred to as a "6 wk old boy" at the outset of the history (line 12) with no other direct references in the rest of the text. As the senior note, the HOSO points to the involvement of "nutrition, card, and SW" (line 15-17). When the "anal" senior resident mentioned above spends 45 minutes editing the Senior Notes on-line, he, in fact, polices this particular indexical system. Much of what he is editing down is previous senior resident's use of "too many" full sentences with pronouns and other direct references to participants. Likewise, medical students are known for writing glaringly long histories. This is partly due to their use of full sentences. Senior residents spend much energy and often abuse to teach interns and medical student how to cut their histories down to the bare minimum. One intern recalled over lunch his preceding surgical rotation where the senior resident insisted 
that the interns' Progress Notes should be no longer than seven lines long and that they should leave space for his note at the bottom of the Progress Note sheet. If not, he would tear up the document.

In summary, we recall that the Senior Note provides a comprehensive mapping of current collaborators and their interdependencies. Interns do not use the HOSO to outline the medical students, senior residents, and the teaching attending physician with whom they are currently working on Pediatric Team B. Yet, when it comes to Dylan and Anna's actual patient history we find little variation in the specification of participants and their relation to the patient - despite the vast difference in length and detail between the senior note and HOSO. For instance, we notice that regardless of the glaring lack of detail in Anna's HOSO history all it misses is a reference to the pulmonary consult involved when she was weaned off diuretics. The senior note does not mention any other participants in the body of the history. Interns would most likely not regard this as an oversight but simply as a fact that is no longer relevant to Anna's current care. In other words, it seems equally important to senior residents and interns alike to index the relationship between caregivers, including themselves, and the individual patients.

\section{Spatial references in the patient history}

In Dylan and Anna's individual histories we find a number of place names - many of which are repeated several times. In the Senior Notes Dylan's history, for instance, mentions the ICU three times, and the floor and shelter two times. Reading those place names within their individual sections we find that they are organized to connote Dylan's trajectory through a number of locales but seen through the lenses of relevant organ systems. The first section summarizing his past and present medical history refers to the ER and the shelter where he lives with his mother. In the next section (i.e., RESP.) we learn that he was transferred to the ICU. The CV section (cardiac vascular) mentions "the floor," Cardio, and F/u in Clinic (follow-up in Clinic). "The floor" refers to his current admission to a general pediatric unit, 10 East. The FEN section refers to ICU, Nutrition, and the floor. The ID section mentions the ICU, and the final section refers to the social worker team and the Shelter. We can depict this general trajectory as follows: Shelter $=>$ $\mathrm{ER}=>\mathrm{ICU}=>$ Floor, Cardiac $=>$ Clinic; $\mathrm{ICU}$, Nutrition $=>$ Floor; ICU $=>$ Social, Shelter. In short, these place names give us a general sense of Dylan's trajectory through the healthcare system from shelter to ER to ICU to floor to follow up care in the clinic and back to the shelter again. Anna's history offers a comparable sequencing of place-names: Common Hospital => ICU => Floor; Pulmonary consult; ICU; ICU; ICU => Discharge pending. We notice that this by no means provides an accurate depiction of her care trajectory; yet, it offers a general sense of her move from Common Hospital transferred to Kiltham's ICU, transferred to the floor and now pending her discharge from the hospital.

The repetition of ICU highlights that most of the significant event took place here. Similarly, the header explicitly states that she is an ICU transfer. To the senior residents this is important information that will prompt their attention. Otherwise, infants with bronchiolitis do not receive much attention during the winter months. The number of admissions with this diagnosis is so high that their care is regarded as routine and something worth little consideration from the senior residents.

The HOSO presents noticeably few placenames compared to the senior note. Anna's case stands out by containing no place names apart from the reference to 10 East in the header. Dylan's history does reference his stay in the ICU and the current involvement of nutrition, cardiac team, social workers, and 
the shelter where his mother lives. However, we do not get a sense of the trajectory spelled out in the senior notes from ER over ICU to floor later to be followed in clinic. Furthermore, there is no repetition of place-names within the history.

The different indexical centering of place names in the two document genres relate closely to their temporal orientation. The trajectories sketched in the Senior Note are a temporal organization of places and participants. In other words, the Senior Note characterizes the relationship between physicians and their object of reference as temporally organized around a sequence of locations involving different participants. In contrast, the HOSO offers a here and now framing of the relation among participants, places, and patient. To better understand those differences in the indexical centering of the patient histories let us elaborate the structure of temporal references used in the two genres.

\section{Temporal references in the patient history}

We find three main types of temporal references in the HOSO and Senior Notes: dates, temporal deictics (e.g., now, recently, currently), and references to the frequency of specific activities (e.g., how often to administrate medication). The header of both HOSO and Senior Notes summarize Dylan and Anna's admission date and their age. The admission date plays an important role in patient care as it pertains to the reimbursement and the physicians' general sentiment of how long time a patient should be in the hospital given the severity of his or her ailment. Frequently, a senior resident or attending doctor will state some variation of the following comment during morning rounds from: "This kid has been here for more than a week. We need to get him rolling." Translated this means that the intern should start working hard on getting the patient ready for discharge.

Dylan and Anna's age can be found just before their chief complaint (e.g. their diagnosis). The location is not random. Physicians regard a four-week-old baby with bronchiolitis very differently than a one-year-old with bronchiolitis. Infants, and in particular prematurely born children, are vulnerable to respiratory diseases and can quickly get gravely sick and require intensive care as in Dylan and Anna's cases. A toddler admitted with bronchiolitis typically stays only a few days. The exact age does not seem to matter a great deal as one often finds variation in the age reported. We notice that the senior note reports Dylan and Anna's ages as one month and five weeks respectively whereas the HOSO gives Dylan's age as 43 days, and Anna's as two months.

We find another set of temporal references in the body of the histories. Marc's HOSO includes dates under the sections, problems and procedures. This builds a temporal field where the date of a procedure, the beginning and end of a problem is the paramount issue at hand. What matters to the interns are when a problem started, or rather was diagnosed, and whether the problem has been resolved. What happens in between does not seem to be essential in the context of the HOSO. The Senior Notes does not demarcate quite as narrow a temporal field. Dylan and Anna's histories contain the dates of several important events. For instance, we learn that Anna was transported from Common Hospital on January 29, intubated in the ICU between January 29 and February 12, and transferred to the floor (i.e., 10 East) on February 15 . Likewise, the senior history provides the dates for a number of different events such as the discontinuation of some of her medication on February 11. In comparison, the HOSO only provides the names of medication currently administrated.

If we introduce temporal deictics into our analysis of the Senior Notes we find that each paragraph builds around a past-present structure. Consider the GI section where I have 
highlighted the temporal deictics, "when" and "now."

GI: On NJ continuous feeds when transferred from ICU. Now on po feeds.

The excerpt follows a past-present structure - explaining that Anna received nutrition through a tube at the time she was transferred from the ICU to 10 East. Now the tube has been removed and she gets her food by mouth. Most of the other paragraphs follow the same structure. The first part of the paragraph summarizes a number of past events and/or test results; a date or temporal deictic typically specifying the timing of the event. The section closes with a description of the current state of affairs, for instance: "now on room air;" or "currently stable."

Dylan's and Anna's histories in the HOSO contain only one such example. In line 12 Dylan's entire hospital trajectory is summarized in one sentence. The remaining sections simply recap the current state of affairs. We learn nothing about past medication or test results. The only other temporal reference we find in Dylan's HOSO history is the frequency by which his medication should be given. Even this is not spelled out very carefully. We learn that he should be given Racemic Epinephrine "when needed" (i.e. PRN). We do not learn how often he should get Azihromycin - only that it should be given for five days. The Senior Notes tend to be more specific, as for instance, in line 16 of Dylan's history: "Started Prosobee at 5cc/hr."

Given that the interns are responsible for patients' medication one might expect that they would record the dose and frequency more carefully in their notes. However, the interns use a separate order sheet and medication chart for this specific purpose, which also serves as a means of communication with the nurses. In other words, giving too much detail on medication in the HOSO would lead to needless repetition. The senior residents do not use the order sheets, so to them it becomes valuable to specify the temporal structure of medication administration.

Comparable to Dylan's case, Anna's HOSO history provides a snapshot of the current state of affairs: Her respiratory distress and bronchiolitis are considered cured on today's date and she receives only medication if needed (i.e., PRN). Anna's HOSO history is a signal to Marc and his fellow interns that Anna is ready to go home and that she requires little if any medical attention. Dylan, in contrast, calls for significantly more involvement and collaboration with several different subspecialties.

\section{Conclusion: Maps \& itineraries}

The HOSO and Senior Note contain many common features and references to participants and their relations to patients. Nevertheless, our analysis reveals important variations in the indexical centering of the two genres. The HOSO builds an indexical ground that offers a here-and-now discursive field for a small group of interns working closely together on a day-today basis. The HOSO does not concern itself with a description of past places and events. It emphasizes the current tasks at hand. Much like an itinerary the HOSO outlines the day's activities facing Marc and his colleagues. The interns have relatively symmetrical access to the knowledge about Anna and Dylan and their past medical history. Every morning they listen to short summaries on their histories.

The interns work in the same team on four to five week rotations. Their collaborators and the departments where their patients are admitted change constantly. Yet, the basic setup remains constant. In contrast, the senior residents' "playing field" can change within one workday when they go from their day work in e.g. Pediatric Team B to covering for other senior residents' teams at night. The senior residents do not share the same degree of symmetrical access to their object of refer- 
ence, the patients. Elisabeth knows a great deal about Dylan and Anna, which she shares with Marc and the other interns. But, at night she covers for other senior residents and must care for patients she knows little or nothing about. While the interns have a high degree of access to each other and a symmetrical access to their object of reference, the senior residents have neither. They do not work shoulder to shoulder with the other senior residents during the day, and they do not see the same patients. With their Senior Notes, the senior residents build a finely marked indexical ground on which they can communicate about their patients. If we look at the first dimension, the relationship between the interacting parties, the Senior Notes containing an explicit structure for the senior residents' current collaborators and the temporal and spatial arrangements under which they work. In terms of the second dimension, the relations between the interacting parties and the patient, the object of reference, histories take into account the lack of relevant knowledge about patients like Dylan and Anna. To account for this lack of symmetrical knowledge the history builds an indexical context that specifies the times and places of the patient's past and present care, tests results, medication, etc. To one of Elisabeth's fellow senior residents on call at night Anna's HOSO history does not make much sense. The HOSO stands out as opaque - maybe even misleading. Apart from Elisabeth, the senior residents do not know what Marc and three other interns know - that Anna, despite the horrible hospital trajectory she has been going through the past few weeks, is set to go home to her mother and father in a day or two. To them Anna's history in the HOSO reads as a patient who has recovered from her bronchiolitis and requires little if any attention. On Marc's part, why should he spend valuable time dissecting the Senior Note to figure out what the current status of Anna's case is when he can simply glance at the HOSO and go to work?
Returning to the questions posed in the introduction, we can now argue that Marc and Elisabeth use different documenting genres as they support differently configured fields of relations to collaborators and patients. Each document genre builds an indexical ground unique to the specific user group. First, these two collective on-line documents summarize two different configurations of collaborators and contemporaries working with an everchanging group of patients. Second, Elisabeth and Marc use the Senior Notes and the HOSO respectively, to demarcate the temporal and spatial structure of their communication practices. When do they have to meet with what people? The use of each document takes place at certain time and places. Elisabeth and Marc share their notes with a different set of collaborators at different times and places. Third, the senior residents and interns build into their information systems indexical structures supporting their unique work practices. The documenting practices involved in the production and use of the HOSO and Senior Note help the interns and senior residents structure where they need to go within the hospital, and in relation to what collaborators and patients.

Those documenting practices structure their use of both time and place. At night Elisabeth typically attends to other patients than Marc in different parts of the hospital, subjects to different temporal rhythms. Furthermore, the two groups do not focus on the same aspects of care. The interns carry out the scut work, and the HOSO gives the times and places where tests should be taken, procedures preformed and patients seen. The senior residents do not go into the same details of care, and their Senior Notes reflect this in the indexical centering of the patient histories. In short, the Senior Notes and HOSO serve two groups requiring different mapping of relationships and itineraries for work practices. As itineraries the two document genres are more than mere lists. The HOSO and Senior Note do not solely map out 
the stable positions among the different interacting parties and their objects of reference. As itineraries they take time and movement into consideration. In de Certeau's words (de Certeau, 1984), the itineraries take into consideration vectors of direction, velocities, and time variables. Each itinerary allows a group to move in a field of interrelated mobile elements.

The importance of a document genre's indexical centering may explain why the field of medical informatics has not succeeded in implementing global patient-centered medical record systems. Such large-scale systems do not allow their users to tailor an indexical field for their interaction. Physicians' resistance to medical informatics, then, cannot be explained by a general technophobia among doctors, as many researches assume, but simply that physicians (and nurses) do not like when somebody messes with the details of their daily itineraries and takes away their ability to fine-tune their collaboration and knowledge sharing with specific constituencies of colleagues.

At this point one could conveniently slip into a polarizing position claiming that all there exists is situated knowledge embedded in the richness of the empirical world. This opposing position would argue that a patientcentered record which attempts to abstract a globally meaningful patient history is all but an impoverished version of the rich and textured situated knowledge held by local participants. However, as Berg (1997a) argues, both positions picture the realm of the abstract and formal as hovering above the realm of the empirical everyday world. "The formal is symbolic, clean, abstract, homogeneous; the empirical is messy, heterogeneous, concrete and not (to be) ordered within one single scheme" (ibid.: 406). One is the global patient-centered record; the other is the lived experience of patients, nurses, and doctors. Berg (ibid.), Markussen (1994), and Star (1995) among others call for reconfiguring this dichotomous opposition between the formal and the informal - the abstract and the situated.

Hanks comes to our rescue. He offers a comprehensive framework demonstrating how abstract and formal deictic systems embedded in linguistic code allow us to situate our everyday communicative practices. To put it differently, the indexical elements embedded in the formal structure of language serve as resources for our evolving practices. They let us situate our practices in complex organizational structures. Through their everyday practices Marc and Elisabeth build indexical fields that permit them to situate their practices across time and many different organizational units involving an ever changing group of participants.

Those linguistic structures embedded in their document genres are windows into the unfolding dynamics of their everyday work practices and their particular position in healthcare.

The Hanks inspired analysis points to a double nature of documenting practices in relation to the doctors' general practices. First, Elisabeth and Marc produce and use the Senior Notes and HOSO as part of their daily communicative practices. The two document genres index these ongoing communicative practices: Who writes what documents? When do they do it? Where to they do it? When and where do they use those documents to structure their discussions about patients? For instance, the first part of the Senior Notes outlines a rudimentary timetable for the production and use of the document and a list of the places and people involved in those documenting practices. Second, Elisabeth and Marc also compose and use the Senior Notes and HOSO to communicate about their ongoing care practices. The HOSO index the involved participants in their daily work whether patients or doctors and nurses. They also demarcate the temporal and spatial structure of those practices. In what departments does Marc have patients at the moment? Where are the 
other collaborators located? What timeframe guide the care? In short, we can argue that the composition and use of the HOSO and Senior Notes allow Elisabeth and Marc to build an indexical structure highlighting the "who, when, and where" of their communicative practices and their general care practices. Documents are both in and about practice. They are both situated and situating. The documents simultaneously make references to and articulate with the context in which the reference is performed.

With the notion of indexical centering in hand we do not have to perceive situated knowledge as merely context-bound. The indexical structures we find in the HOSO and Senior Notes demonstrate that physical boundaries such as an inpatient unit or the emergency room do not define the situated field within which Marc and Elisabeth operate. The two doctors use their documenting practices to situate their work in complex cross-contextual relations involving many different locales, professional groups, patients, and relatives. With this in mind we can reformulate the problem of distributed knowledge. The issue is not so much how or whether people share situated knowledge across boundaries as how they use documents to demarcate the "who, when, and where" for their knowledge sharing.

Indexical analysis of documenting practices can inform our understanding of how information systems support various configurations of distributed and virtual work as it addresses the temporal and spatial organization of work among a group of participants. Attempts to support distributed collaboration and the asymmetrical access to knowledge involved may gain from studying the indexical structures of, for instance, the Senior Notes supporting such distributed organizational realities. Likewise, faceto-face work, or combinations of co-located and distributed work, could call for an indexical centering comparable to the HOSO. In short, I believe that a further refinement of Hanks' general approach to communicative practices will inform our approach to knowledge sharing in organizations and the way information systems support such practices. Equally important, such a focus would allow us to re-conceive the dream of the universal patient-centered record and help the field of medical informatics build systems that better serve doctors' and nurses' daily care for patients and the organizational realities they face.

\section{References}

Bakhtin, M. M. (1986). "The Problem of Speech Genres." In Speech Genres and Other Late Essays: M. M. Bakhtin, C. Emerson and M. Holquist (ed.), pp. 60-102. Austin: University of Texas Press.

Berg, M. (1997a). "Of Forms, Containers, and the Electronic Medical Record: Some Tools for a Sociology of the Formal." Science, Technology, \& Human Value 22: 403-33.

Berg, M. (1997b). Rationalizing Medical Work: Decision-Support Technologies and Medical Practices. Cambridge, MA: MIT Press.

Berg, M., Aarts J., van der Lei, J. (2003). "ICT in Health Care: Sociotechnical Approaches." Methods of Information in Medicine 42: 297-301.

de Certeau, M. (1984). The Practice of Everyday Life. Berkeley: University of California Press.

Dreier, O. (1999). "Personal Trajectories of Participation Across Contexts of Social Practice." Outlines: Critical Social Studies 1: 5-32.

Epstein, J. (1995). Altered Conditions: Disease, medicine, and storytelling. New York: Routledge.

Grandori, A., Kogut, B., Lewin, A., (ed.) (2002). "Knowledge, Knowing, and Organizations." Organization Science, 13 (3).

Hanks, W.F. (1990). Referential Practice: Language and lived space among the Maya. Chicago: University of Chicago Press.

Hanks, W.F. (1996). Language \& Communicative Practices. Boulder: Westview Press. 
Hanks, W.F. (2000). Intertexts: Writings on language, utterance, and context. Lanham, MD: Rowman \& Littlefield.

Ito, M. (1999). "Network Locality." Presented at Society for the Social Studies of Science meetings, San Diego.

Ito M. (2001). "Mobile Phones, Japanese Youth, and the Re-placement of Social Contact." Presented at Society for the Social Studies of Science meetings, Boston.

Markussen, R. (1994). "Dilemmas in Cooperative Design.” Presented at PDC '94: Proceedings of the Participatory Design Conference, Palo Alto.

Merriam-Webster's Online Dictionary (2001) "Deictic". In Encyclopedia Britannica Online. (http://80-www.search.eb.com.libezproxy.syr. edu/dictionary

Orlikowski, W.J. (2002). "Knowing in Practice: Enacting a collective capability in distributed organizing." Organization Science 13: 0249-73.

Østerlund, C.S. (2002). Documenting Dreams: Patient-Centered Records versus PracticeCentered Records. Unpublished Ph.D. Dissertation. M.I.T., Cambridge. poi-wg@mail.amia.org. AMIA PEOPLE AND ORGANIZATIONAL ISSUES WORKING GROUP.

Polanyi, M. (1983). The Tacit Dimension. Gloucester, Mass.: Peter Smith.

Star, S.L. ed. (1995). Ecologies of Knowledge: Work and Politics in Science and Technology. New York: State University of New York Press.

Timmermans, S., Berg, M. (2003). The Golden Standard: The challenge of Evidence-based medicine and standardization in health care. Philadelphia: Temple University Press.

Walsh, J.P. (1995). "Managerial and organizational cognition: Notes from a trip down memory lane." Organization Science 6: 280-321.

Walsh, J.P, Ungson, G.R. (1991). “Organizational Memory." Academy of Management Review 16: 57-91.

Weed, L. (1968). "Medical records that guide and teach." New England Journal of Medicine 278: 652-57.

Zerubavel, E. (1979). Patterns of Time in Hospital Life. Chicago: The University of Chicago Press. 